\title{
Médiévales
}

Langues, Textes, Histoire

57 | automne 2009

Langages politiques, $\mathrm{XII}-\mathrm{XV}$ siècle

\section{Le conseil de Jéthro à Moïse: le rebond d'un fragment de théologie politique dans la rhétorique parlementaire castillane}

Moses' Advice to Jethro: Reviving a Fragment of Political Theology in Castilian

Parliamentary Rhetoric

\section{François Foronda}

\section{OpenEdition \\ Journals}

Édition électronique

URL : https://journals.openedition.org/medievales/5805

DOI : 10.4000/medievales.5805

ISSN : 1777-5892

Éditeur

Presses universitaires de Vincennes

Édition imprimée

Date de publication : 20 décembre 2009

Pagination : 75-92

ISBN : 978-2-84292-241-2

ISSN : 0751-2708

\section{Référence électronique}

François Foronda, «Le conseil de Jéthro à Moïse: le rebond d'un fragment de théologie politique dans la rhétorique parlementaire castillane », Médiévales [En ligne], 57 | automne 2009, mis en ligne le 18 janvier 2012, consulté le 22 avril 2022. URL : http://journals.openedition.org/medievales/5805 ; DOI : https://doi.org/10.4000/medievales.5805 


\section{LE CONSEIL DE JÉTHRO À MOÏSE : \\ LE REBOND D'UN FRAGMENT DE THÉOLOGIE POLITIQUE DANS LA RHÉTORIQUE PARLEMENTAIRE CASTILLANE}

Alors qu' Israël vient de dresser son campement aux pieds du Sinaï et s'apprête à négocier sa loi avec Dieu, Moïse reçoit la visite de son beaupère, Jéthro, prêtre de Madian (Ex. 18, 1-12). Le lendemain, Jéthro voit son gendre tenir audience du matin au soir. Il le voit ainsi s'épuiser et épuiser son peuple aussi, contraint d'en venir jusqu'à lui seul pour obtenir un jugement. Pour les soulager l'un et l'autre, Jéthro conseille à Moïse de ne plus se soucier que des choses supérieures et de déléguer à d'autres le soin des affaires courantes. Moïse désigne alors les juges d'Israël (Ex. 18, 13-27), ouvrant une possibilité de diffusion et de réappropriation de la loi avant même qu'elle ne se trouve fixée ${ }^{1}$.

Amplification midrashique des paroles par lesquelles Moïse pose aussi d'une certaine manière le principe de séparation des pouvoirs $(\text { Dt } 1,9-18)^{2}$, qui ne rencontre cependant pas d'écho particulier dans l'exégèse biblique au Moyen Âge ${ }^{3}$, ce conseil de Jéthro fait irruption en 1385 puis en 1469 dans une rhétorique parlementaire castillane qu'une forte crise de régime - dont nous verrons au fur et à mesure les différents contextes - pousse dans les

1. F. OsT, «Le Sinaï ou la loi négociée », dans son ouvrage Raconter la loi. Aux sources de l'imaginaire juridique, Paris, 2004, p. 68.

2. J. van SETERS, «Etiology in Moses Tradition : the Case of Exodus 18 », Hebrew Annual Review, 9, 1985, p. 355-361 ; B. M. Levinson, « The First Constitution : Rethinking the Origins of Rule of Law and Separation of Powers in Light of Deuteronomy », Cardozo Law Review, 27/4, 2006, p. 1853-1888.

3. Ph. Buc, L'ambiguïté du Livre. Prince, pouvoir, et peuple dans les commentaires de la Bible au Moyen Âge, Paris, 1994. 
deux cas en direction du sermon politique ${ }^{4}$. Mais si en 1385 , ce fragment de théologie politique est employé par Jean $\mathrm{I}^{\text {er }}(1379-1390)$ pour instituer le Conseil, et calmer ainsi le remous produit dans les rangs des représentants des villes par la défaite d'Aljubarrota contre le Portugal ${ }^{5}$, c'est une autre génération de ces représentants qui s'en empare en 1469, au terme d'une guerre civile, pour rappeler à Henri IV (1454-1474) l'origine du contrat tacite (contrato callado) qui le lie au royaume et l'oblige à un certain exercice du gouvernement ${ }^{6}$.

Un rebond discursif et critique a donc lieu en 1469. Il atteste de la réception préalable du message émis en 1385, mais aussi de sa compréhension particulière par une composante de la société politique - les villes, leurs représentants, leurs oligarchies gouvernantes - qui réclame de participer à cette «monarchie nobiliaire» qui se met en place dans la Castille de la fin du Moyen Âge par la privanza (privauté), c'est-à-dire un système politique fondé sur la faveur, engageant de manière expansive les élites politiques dans le rehaussement souverain ${ }^{7}$. Deux questions se trouvent donc intimement mêlées : la circulation d'un message et le partage du pouvoir, la première étant à la fois le reflet et le vecteur du second.

Entre ces deux emplois du conseil de Jéthro, un troisième se produit, chronologiquement le deuxième, qui signale qu'un relais s'est probablement opéré au cours des années 1430, créant les conditions du rebond discursif et critique de 1469, mais en marge de la scène parlementaire. Il s'agit, dans la continuité de l'emploi de 1385, de celui qu'en fait l'observantin Juan de

4. Pour la péninsule Ibérique, les études consacrées à la rhétorique parlementaire envisagent principalement le cas de la couronne d'Aragon : P. M. CÁTEDRA, Dos estudios sobre el sermón político en la España medieval, Barcelone, 1981 ; ID., " Acerca del sermón político en la España medieval (a propósito de Martín el Humano en las Cortes de Zaragoza de 1398) », Boletín de la Real Academia de Buenas Letras de Barcelona, 40, 1985-86, p. 17-47 ; M. D. Johnston, «Parliamentary oratory in medieval Aragon », Rhetorica, A Journal of the History of Rhetoric, 10 (2), 1992, p. 92-117 ; S. F. CAWSEY, « Royal eloquence, royal propaganda and the use of the sermon in the medieval Crown of Aragon, ca 1200-1410», Journal of Ecclesiastical History, 50 (3), 1999, p. 442-463 ; ID., Kingship and Propaganda: Royal Eloquence and the Crown of Aragon, ca 1200-1450, Oxford, 2002.

5. Valladolid, 1385, Cortes de los antiguos reinos de León y de Castilla (CLC), Madrid, 1863 (1866 et 1882 pour les t. III et IV), t. II, p. 333-335. Une version quelque peu distincte de ce razonamiento est consultable en ligne, à partir de son édition dans F. MARTínEZ MARINA, Teoría de las Cortes o Grandes Juntas Nacionales, Madrid, 1813 (http://www.cervantesvirtual.com/ servlet/SirveObras/13594952101248274198835/p0000011.htm\#I_88_).

6. Ocaña, 1469, CLC, III, p. 767-769. Le texte est repris dans l'article de R. MoRán MARTín, «Alteza... merçenario soys. Intentos de ruptura institucional en las Cortes de León y Castilla », dans Coups d'État à la fin du Moyen Âge? Aux fondements du pouvoir politique en Europe occidentale, F. Foronda, J.-Ph. GENET et J. M. Nieto SoRIA dir., Madrid, 2005, p. 108-112.

7. F. Foronda, La privanza ou le régime de la faveur. Essai sur la souveraineté dans la Castille de la fin du Moyen Âge, Madrid, à paraître. Sur l'intégration urbaine au régime de privanza, voir M. ASENJo GoNZÁLEZ, «La aristocratización política en Castilla y el proceso de participación urbana (1252-1520) », dans La monarquía como conflicto en la Corona castellanoleonesa (ca 1230-1504), J. M. NiETo SORIA dir., Madrid, 2006, p. 133-196. 
Alarcón dans Libro del regimiento de los señores qu'il dédie vers 1436 au principal privé (privado) de Jean II (1406-1454), don Álvaro de Luna ${ }^{8}$.

Trois temps se dégagent par conséquent, qui seront ceux de cette étude, celui de l'invention d'abord, du relais ensuite, de la récupération enfin. Cette scansion dans le rebond du conseil de Jéthro, finalement transformé en récit fondateur d'un gouvernement en partage ${ }^{9}$, signale au-delà les temps d'un processus d'alphabétisation politique, et par conséquent d'intégration, qui fait que le roi, ses officiers et les représentants des villes parlent en définitive un même langage politique.

\section{L'invention}

L'arrivée sur le trône des Trastamare en 1369 ouvre en Castille un nouveau cours gouvernemental. Il répond à l'attente qu'ils ont eux-mêmes avivée au sein de la société politique pendant la guerre civile, essentiellement celle d'un gouvernement mieux réglé et donc plus transparent, plus ouvert aussi. Cette glasnost est principalement marquée par l'institutionnalisation des principaux corps de l'État (la chancellerie et le tribunal de l'Audience en 1371, le Conseil royal en 1385) et la réorganisation du service au roi, du dehors d'abord (la connétablie en 1382), du dedans ensuite (la chambre des pannes en 1385) ${ }^{10}$. Si cette suite de décisions a conduit à supposer l'existence d'un programme de réforme ${ }^{11}$, de modernisation étant donné l'aveu pour certains de ses volets de mettre le royaume de Castille au diapason des autres grands royaumes occidentaux ${ }^{12}$, force est de constater que le point d'orgue dudit programme, l'institution de cette pratique jusque-là très régulière mais informelle de gouvernement qu'est le Conseil, n'y semblait pas figurer au départ. Car loin de représenter l'acte souverain d'un pouvoir triom-

8. El libro del regimiento de los señores de fray Juan de Alarcón, M. C. Pastor CuEvas éd., Madrid, 2000, I.8 et III.4, p. 259-260 et 307-309. Le texte est consultable en ligne à partir d'une édition plus ancienne (Libro del regimiento de los señores, F. RuBio éd., dans le t. II des Prosistas castellanos del siglo XV, vol. 171 de la Biblioteca de Autores Españoles, Madrid, 1964) sur le site de la Biblioteca Saavedra Fajardo de Pensamiento Político Hispánico de l'Université de Murcie (saavedrafajardo.um.es).

9. Voir en ce sens les remarques de N. JEAMMET, qui insiste sur le projet communautaire à inventer contenu dans le Nom transmis à Moïse, lequel se soumet à une loi de mutualité en acceptant de désacraliser son pouvoir en le partageant avec d'autres ( La double valence de la culpabilité », L'Esprit du Temps, 19, 2007, p. 177-178).

10. L. V. DíAz MARTín, Los orígenes de la Audiencia real castellana, Séville, 1997 ; C. GARRIGA, La Audiencia y las Chancillerías castellanas (1371-1525), Madrid, 1994 ; S. DE Dios, El Consejo Real de Castilla (1385-1522), Madrid, 1982.

11. L. SuÁREZ FERnáNDEZ, Historia del reinado de Juan I de Castilla, t. I : Estudio, Madrid, 1977.

12. Outre le razonamiento, voir la création de l'office de connétable en 1382 ou encore l'abandon de l'ère hispanique en 1383 (ibid., t. II : registro documental (1371-1383), Madrid, 1982 , pièces $\mathrm{n}^{\text {os }} 307$ et 367 , p. 444-446 et 548-553). 
phant, l'institution du Conseil royal signale plutôt la repentance d'une royauté quelque peu chancelante.

Cette situation tient à la défaite militaire d'Aljubarrota, le 14 août 1385, contre le Portugal dont Jean $\mathrm{I}^{\mathrm{er}}$ ambitionne de porter la couronne. Elle ouvre une crise de confiance sans précédent, seize ans seulement après la victoire d'Henri II (1369-1379), et rend plus offensive la réclamation de la couronne castillane par le duc de Lancastre, Jean de Gand, en vertu de son mariage avec Constance de Castille, fille de Pierre le Cruel (1350-1379). C'est donc pour rétablir la confiance que Jean $\mathrm{I}^{\text {er }}$ en vient à instituer le Conseil royal, sa chancellerie montant à cette occasion une opération de communication politique jusque-là inédite, sous la forme d'un razonamiento, plus une harangue qu'un strict sermon en fait, que le roi prononce dans la cathédrale de Valladolid, lors de la dernière des séances solennelles des Cortes, le $1^{\text {er }}$ décembre $1385^{13}$.

Le roi y précise les quatre raisons l'ayant conduit à ordonner ce qui est explicitement présenté comme une innovation institutionnelle. La première concerne la guerre: le roi veut pouvoir s'y consacrer entièrement, aussi envisage-t-il de déléguer son autorité afin de ne pas avoir à s'occuper de tout. La deuxième concerne la rumeur : contre ces dires qui l'accusent de n'en faire qu'à sa tête et sans conseil, il prévoit de choisir certains hommes - quatre membres du clergé, quatre de la noblesse et, innovation peut-être plus fondamentale encore que cette institution même, du moins c'est là sa pierre d'angle, quatre des villes ${ }^{14}$ - pour qu'ils examinent les affaires du royaume. La troisième répond à la demande de participation des Cortes, en particulier au sujet des demandes financières qu'elles estiment exagérées et qu'elles souhaitent pouvoir contrôler. La quatrième raison, déclarée la principale, porte sur la maladie du roi. Tous ont pu la constater en raison des nombreuses rechutes qu'il a endurées, et le légitime souci qu'il a pour sa santé l'empêche de répondre personnellement à toutes les suppliques.

C'est donc un roi de souffrance que découvrent les représentants des villes aux Cortes de Valladolid, qui n'accepte d'ailleurs de ne tenir audience qu'une fois par semaine, sans précision du jour ${ }^{15}$. Remarquons que la souffrance avouée du corps se double d'une autre, plus intime, celle du cœur,

13. Valladolid, 1385, CLC, II, p. 333-335.

14. Les villes tentent dès le début de la « révolution» trastamare d'obtenir une représentation permanente dans un Conseil encore non institué mais qu'elles savent cependant stratégique (Burgos, 1367 et Toro, 1369, ibid., p. 148 et 183). En 1371, Henri II leur oppose une fin de non-recevoir au prétexte qu'il peut compter désormais sur les auditeurs d'une Audience tout juste instituée, soit des officiers plutôt que des représentants (Toro, 1371, ibid., p. 211).

15. Valladolid, 1385, ibid., p. 329. Sur le rituel de l'audience publique, voir mes études «La propagande monarchique dans la Castille du XIII ${ }^{\mathrm{e}}$ siècle. Considérations autour du Libro de los doze sabios », dans Convaincre et persuader. Communication et propagande aux $\mathrm{XII}^{e}$ et XIII ${ }^{e}$ siècles, M. Aurell dir., Poitiers, 2007, p. 279-299 ; «Las audiencias públicas de la reina Isabel en Sevilla (1477) : ¿La resorción administrativa de un improbable ritual de gobierno ?», dans Gobernar en tiempos de crisis. Las quiebras dinásticas en el ámbito hispánico, J. M. NIETO SORIA et M. V. LóPEZ CORDÓN dir., Madrid, 2008, p. 133-171. 
dont l'affliction s'exprime au-dehors par les vêtements de deuil que porte le roi, pour n'avoir pas tenu assez bien sa justice, pour avoir imposé à son peuple les trop lourdes charges de la guerre, pour être encore obligé de demander davantage plutôt que d'alléger ses exigences, pour les pertes enfin d'une guerre que la défaite a transformé en déshonneur ${ }^{16}$. Aljubarrota force donc le roi à faire contrition, dans l'espoir que Dieu éteigne sa colère et accorde la victoire. Pour finir d'obtenir sa miséricorde, le roi donne d'ailleurs à son peuple la possibilité de communier avec lui dans une même repentance en ordonnant au clergé de son royaume qu'il organise des processions, des prêches et des jeûnes ${ }^{17}$. C'est ce contexte de contrition qui invite à rechercher plus au-dedans d'une certaine manière les raisons de l'emploi du conseil de Jéthro à la suite de la quatrième et principale raison du razonamiento.

Ce conseil pose donc l'analogie entre la souffrance royale et l'épuisement mosaïque, justifiant ainsi une institution politiquement apaisante. Secondairement, elle est peut-être destinée à séduire au-delà même du royaume. C'est vers la France et l'Aragon que se sont principalement tournés les historiens soucieux d'identifier les modèles ayant pu inspirer à Jean $\mathrm{I}^{\mathrm{er}}$ cette institution du Conseil. Il convient cependant de donner plus de crédit à la piste portugaise. En raison de la revendication de ce royaume par Jean $\mathrm{I}^{\mathrm{er}}$ d'abord, le roi s'obstinant en effet dans ce dessein après Aljubarrota, comme le montrent certaines des considérations de son razonamiento de 1385 et plus encore le fait qu'il en vienne plus tard, en 1390, à envisager très sérieusement d'abdiquer en faveur de son fils Henri pour donner plus de force à sa revendication auprès de l'opinion portugaise. Ensuite, la piste portugaise est à considérer en raison d'une certaine convergence entre les demandes de participation gouvernementale qu'expriment les conseils urbains portugais aux Cortes de Coimbra, en avril 1385, celles de la proclamation royale du maître d'Avis, et les revendications dans ce même sens des représentants des villes castillanes aux Cortes de Valladolid, seulement quelques mois plus tard. Car tant aux Cortes de Coimbra comme à celle de Valladolid, la réponse donnée à ces revendications consiste notamment en une « création » du Conseil qui revient à en régler l'ouverture à cette composante sociale ${ }^{18}$.

Apaiser et séduire, reprendre ainsi la main, voici en somme ce qui gouverne l'invention du conseil de Jethro. Ses inventeurs n'ont pas eu à chercher bien loin en fait. En effet, parmi les matériaux que la Chancellerie trastamare utilise pour rénover et étoffer ses pratiques préambulaires, jusqu'au point parfois de gonfler certains des actes les plus significatifs de la «révolution trastamare » à partir de 1379 par des sortes d'accessus ${ }^{19}$, se trouve

16. Valladolid, 1385, CLC, II, p. 330-331.

17. Ibid., p. 332.

18. A. DE SousA, «O discurso político dos concelhos nas cortes de'1385», Revista de Facultade de Letras. Historiá, 2, 1985, p. 9-44.

19. Outre l'acte déjà indiqué portant création de la connétablie, qui insiste tout particulièrement sur les vertus attendues du plus grand des officiers du roi et que signe un Alvarus decretorum doctor (il s'agit d'Álvaro, évêque de Zamora en 1377 et conseiller du roi en 1381), voir le développement sur la loyauté dans la concession de la seigneurie de Melgar à Pedro Fernández 
notamment le Tratado de la comunidad, composé très probablement alors, qui est en réalité une traduction partielle du Communiloquium de Jean de Galles, soit de l'une des plus importantes batteries d'exempla en usage dans l'Occident de la fin du Moyen Âge ${ }^{20}$. Et sans doute la forte présence d'ecclésiastiques à la Chancellerie royale, notamment de franciscains, à la cour des Trastamare ${ }^{21}$, explique-t-elle la traduction de ce texte à l'origine déjà d'une partie des gloses ajoutées par fray Juan de Castrojeriz au De regimine principum, que cet autre franciscain avait traduit en castillan pour l'instruction de Pierre le Cruel quand il était infant, mais qui s'était finalement converti en la bible doctrinale de ses opposants ${ }^{22}$. Tant dans la Glosa que dans le Tratado $^{23}$, en raison de cette communauté de source ${ }^{24}$, le conseil de Jéthro sert sommairement à rappeler au roi l'indispensable conseil qu'il lui faut demander pour gouverner, y compris à des plus «petits » et à des hommes moins sages que lui. On est certes bien loin du long développement du même conseil dans le razonamiento, mais il faut souligner entre ce dernier et les précédents la concordance dans l'intention d'une part, soit l'ouverture du Conseil, et d'autre part le probable rôle d'aiguillage qu'ont pu remplir à partir de là ces mentions en direction d'autres sources au récit plus étoffé.

Bien entendu le razonamiento indique comme source l'Écriture Sainte. Toutefois la narrativité du passage, voire son caractère didactique, oblige à se tourner non pas tant en direction de la Bible que d'un texte dont la transmission manuscrite montre qu'il en a fait office dans la Castille du bas Moyen Âge, pour l'Ancien Testament essentiellement, en vulgaire et de manière historiée : la General Estoria d'Alphonse X (1252-1284) ${ }^{25}$. Annoncé à l'occa-

Cabeza de Vaca en 1379 (L. SuÁRez Fernández, Historia del reinado de Juan I, op. cit., t. II, pièce $\mathrm{n}^{\circ}$ 56, p. 61-62).

20. Tratado de la comunidad (Biblioteca de El Escorial, ms. \&-II-8), F. A. RAMíREZ éd., Londres, 1988). Sur les sources de ce traité, voir C. WitTLin, "Footnote to a book review », La Corónica, 18 (2), 1990, p. 128-129 et C. GuARdiola, « Observaciones sobre la fuente del Tratado de la comunidad », Anuario medieval, 3, 1991, p. 138-148. Et sur l'influence de Jean de Galles en Castille: A. M. HuÉlamo SAn José, «El Communiloquium de Juan de Gales en las letras castellanas », dans J. M. LuCíA Megías (coord.), Actas del VI Congreso Internacional de la Asociación hispánica de Literatura Medieval, Alcalá de Henares, 1997, t. II, p. 821-828.

21. J. M. NieTo SoRIA, «Franciscanos y franciscanismo en la política y en la corte de la Castilla Trastamara (1369-1475) », Anuario de Estudios Medievales, 20, 1990, p. 109-131 ; ID., Iglesia y génesis del Estado moderno en Castilla (1369-1480), Madrid, 1994, p. 165-171.

22. F. Gómez Redondo, Historia de la prosa medieval castellana, t. II : El desarollo de los géneros. La ficción caballeresca y el orden religioso, Madrid, 1999, p. 1704-1725.

23. Glosa castellana al "regimiento de Príncipes » de Egidio Romano, J. BENEYTo PÉREZ éd., Madrid, 1947, t. III, p. 189 (réimpr. Madrid, 2005, p. 849) ; Tratado de la comunidad, op. cit., p. 110.

24. Communiloquium I.6.7 (Summa de regimine vite humanae seu margarita doctorum ad omne propositum, Venise, 1496), d'après la table proposée par C. GuARDiola, loc. cit., p. 143.

25. M. Morreale, «La General estoria de Alfonso X como Biblia », dans G. Bellini (coord.), Actas del Séptimo Congreso de la Asociación Internacional de Hispanistas, Rome, 1982, t. I, p. 767-773 ; P. SÁNCHEZ-PRIETO BoRJA, «Biblias romanceadas », dans Diccionario filológico de literatura medieval española. Textos y transmisión, C. AlvaR et J. M. LuCía MEGías dir., Madrid, 2002, p. 212-223. 
sion du chapitre consacré aux réjouissances pour la victoire d'Israël contre Amalech ${ }^{26}$, le conseil de Jéthro à Moïse occupe ensuite pas moins de deux chapitres ${ }^{27}$. Le premier relate le conseil proprement dit et précise le principe d'organisation des magistratures (chiliarques, centurions, cinquanteniers, décurions), tandis que le second, plus original par rapport aux sources employées dans ces mêmes chapitres, les Antiquités judaïques de Flavius Josèphe et surtout l'Historia scholastica de Pierre le Mangeur ${ }^{28}$, indique la mise en place d'un échelon judiciaire intermédiaire, des juges d'appel en somme, cet échelon étant destiné à assurer un dernier filtrage avant que les affaires véritablement les plus importantes ou les cas les plus graves ne parviennent à Moïse. Faut-il interpréter ce rendu du conseil de Jéthro dans la General Estoria comme le signe d'une recherche de modèles ou de justifications au moment où les Cortes de Zamora de 1274 obligent ce roi-législateur qu'est Alphonse $\mathrm{X}$ à recentrer son ambition politique sur la seule figure du roi-juge ${ }^{29}$, en consacrant sa majorité de justice (mayoría de justicia), en établissant des juges de cour et en définissant les cas royaux ? Quoi qu'il en soit, l'inscription du conseil de Jéthro dans cette sorte de majorat idéologique dont fait partie la General Estoria dès sa constitution par Alphonse X rend pour le moins hasardeuse l'affirmation de Salustiano de Dios, lequel estime cette référence tout à fait étrangère à la réalité castillane ${ }^{30}$. Et c'est en relation avec ce patrimoine référentiel, que certains rois œuvreront à augmenter, qu'il faut chercher à comprendre la réactivation de 1385.

Il convient de remarquer que le très rapide réarmement des ambitions politiques alphonsines après leur échec, en passe sous le règne de Sanche IV (1284-1295) par un aggiornamento dans le sens d'une monarchie sacerdotale, qui prend acte d'une certaine manière du bougé politique intervenu en 1274 à Zamora. Idéologiquement, elle prend principalement appui sur un modèle mosaïque, comme le montre par exemple la représentation du roilégislateur dans le manuscrit enluminé de la première des Partidas que réalise l'atelier royal au début des années $1290^{31}$, ou encore le rêve que Sanche IV fait d'un roi-juge en majesté dans ses Castigos en 1292-1293, lequel trône dans une salle d'audience inspirée de la Tente de la Rencontre et porte de lourds habits scintillants de vertus semblables aux vêtements sacerdotaux

26. Alfonso el Sabio, General Estoria, P. SÁnchez-Prieto Borja éd., Madrid, 2001, t. II, Première partie, L. XIV, chap. 9, p. 214-215.

27. Ibid., Première partie, L. XIV, chap. 10 et 11, t. II, p. 391-393.

28. Ces sources sont précisément mentionnées dans les chapitres ci-dessus indiqués. Voir Flavius JosèPhe, Antiquités judaïques, trad. J. Weill, Paris, 1900, t. I, L. III, chap. 4, p. 159160 ; Petrus Comestor, Scolastica historia, Chambéry, 1485, non folioté (Super exodi libro, De subdiuisione magnatum ad concilium ietro).

29. A. Iglesia Ferreirós, «Las Cortes de Zamora de 1274 y los casos de Corte », Anuario de derecho del derecho español, 41, 1971, p. 945-971.

30. S. DE Dios, El Consejo Real, op. cit., p. 75.

31. F. ForondA, «Le Verbe législatif alphonsin. Hypothèses de lecture de quelques miniatures du manuscrit Add. 20787 de la British Library », e-Spania. Revue électronique d'études hispaniques médiévales, 4, 2007 (http://e-spania.revues.org/document1703.html). 
d'Aaron ${ }^{32}$. Dans ce rêve, le roi-juge, dans l'attente que quelque sujet vienne l'aviver dans sa majesté par sa supplique, n'est pas seul. Deux serviteurs et les douze hommes d'honneur de son conseil l'entourent, l'identité de ces derniers restant indéfinie, leur fonction dans l'accomplissement du devoir d'écoute du roi assez indécise aussi. Remarquons que près d'un siècle plus tard, ce sont précisément ces points que règle Jean $\mathrm{I}^{\mathrm{er}}$, en désignant les douze hommes avec le souci d'égaliser la représentation du clergé, de la noblesse et des villes dans ce groupe, et en précisant le champ de leur compétence, seuls les cas relevant de son Audience et ce qu'il juge être son domaine réservé, principalement l'administration de sa faveur, se trouvant exceptés ${ }^{33}$.

Autrement dit, si la Chancellerie exhume en 1385 le conseil de Jéthro, c'est autant pour réaffirmer l'actualité du projet sanchiste de monarchie sacerdotale que pour procéder à sa révision. Celle-ci légitime et légalise un gouvernement en partage de fait déjà en vigueur, mais pour la première fois officiellement ouvert aux villes en tant que telles, tout en consacrant la supériorité souveraine du guide. En somme, le conseil gouverne et le roi règne. Toutefois, la victoire remportée par les villes en 1385 n'est que de courte durée. Deux ans plus tard, aux Cortes de Briviesa, où Jean $\mathrm{I}^{\mathrm{er}}$ abandonne enfin le deuil qu'il portait depuis $1385^{34}$, aux villes qui tentent de pousser encore et trop leur avantage en lui réclamant cette fois d'exclure les Grands de son Conseil, il coupe court en rappelant le principe de libre élection de ses conseillers, ce qui exclut une représentation permanente des villes en tant que telles à son conseil, mais non pas leur présence qui, du point de vue royal, reste de toute façon assurée par les docteurs et les letrados ${ }^{35}$. L'heure

32. Castigos del rey don Sancho IV, H. Ó. BIZZARRI éd., Madrid, 2001, chap. XI, p. 142151. Signalons dans ces Castigos, l'insertion d'un autre récit de souveraineté - réélaboré en 1353 dans le sens d'une plus nette articulation entre celle-ci et la privanza-, celui du concours des gardes du corps de Darius (remplacé ici par Zorobabel), dont la source signalée est un livre (apocryphe) d'Esdras qui présente une Septante originale en raison précisément de l'inclusion de ce récit. C'est là une haggada liée à la tradition populaire du misdrach, qui réélabore un récit de propagande achéménide lui-même inspiré d'un mythe indo-iranien de refondation cosmique de la royauté (ibid., chap. XXXIII, p. 245-247 et n. 15 aux mêmes pages ; F. ZIMMERMAN, «The Story of the Three Guardsmen », The Jewish Quaterly Review, New Series, 54/3, 1964, p. 179-200 ; A. Hilhorst, « Darius' Pillow (I Esdras iii.8)», Journal of Theological Studies, 33, 1982, p. 161-163 ; A. CANESSA, "Le concours des gardes du corps de Darius dans Esdras A' », Pallas, 44, 1996, p. 25-45). Avec le conseil de Jéthro, ce récit pointe par conséquent une lointaine veine midrashique dans le discours politique castillan ; la spécificité de ce dernier dans le contexte européen du XIII ${ }^{\mathrm{e}}$ siècle étant par ailleurs son orientalisme, particulièrement net dans les miroirs aux princes de l'époque alphonsine. Sur ce dernier point, voir A. RucQuor et H. Ó. BIzZARri, «Los Espejos de Príncipes en Castilla : entre Oriente y Occidente », Cuadrenos de Historia de España, 79, 2005, p. 7-30.

33. Valladolid, 1385, CLC, II, p. 332-333.

34. Briviesca, 1387, ibid., p. 398.

35. Ibid., p. 382 . Il faut par la suite attendre 1425 pour que les villes réclament à nouveau une représentation permanente au Conseil (Palenzuela, 1425, CLC, III, p. 56). De récentes analyses montrent qu'elles obtinrent peut-être satisfaction à partir de cette date, mais au prix d'une drastique réduction de leur présence à des Cortes - alors que les villes y étant représentées étaient au nombre de 100 en 1315, elles ne sont plus que 49 en 1391 puis 17 sous les règnes 
n'est plus à la repentance mais bien à la reprise en main d'un corps de l'État que le roi n'avait consenti de séparer formellement de son simple corps épuisé et souffrant que pour régler une crise de confiance.

\section{Le relais}

Sur la scène parlementaire, plus personne ne parle du conseil de Jéthro jusqu'en 1469. Mais une trentaine d'années avant qu'il n'y rebondisse, en marge de celle-ci, frère Juan de Alarcón y prête une attention soutenue, laquelle doit être envisagée depuis la perspective de la communication royale en direction des villes. Avec quelques nuances cependant par rapport à la situation de 1385, qui forcent à relativiser cette idée de "communication ». Car ce n'est plus le roi qui communique mais son privé, don Álvaro de Luna, qui suscite la rédaction, par un homme qu'il aide à implanter l'Observance en Castille, d'un traité dénué d'une quelconque portée à en juger par sa tradition manuscrite ${ }^{36}$. L'insertion du conseil de Jéthro dans cette réflexion principalement axée sur la responsabilité des gouvernants ${ }^{37}$, que Dieu laisse toujours libres de suivre leur propre mouvement ${ }^{38}$, démontre néanmoins une tentative de conciliation, entre d'une part les positions défendues par les tenants $\mathrm{du}$ gouvernement luniste contre les parents du roi, les Infants d'Aragon, et leurs partisans, et d'autre part celles qu'expriment les représentants des villes aux Cortes, dont la «fonctionnarisation » s'amorce alors à marche forcée ${ }^{39}$. Et cette évolution explique sans doute pour partie le saut qualitatif qu'enregistre au cours des années 1430-1440 leur rhétorique pétitionnaire.

Aussi, faut-il voir dans cette tentative de conciliation un aspect du versant « urbain » de la politique de capture sociétale engagée par don Álvaro pour se maintenir au pouvoir, laquelle articule déjà une forte pénétration dans les rangs de la noblesse seconde et une promotion décisive de ces letrados dont Jean $\mathrm{I}^{\text {er }}$ affirmait en 1387 qu'ils représentaient les villes à son Conseil ${ }^{40}$.

de Jean II et d'Henri IV, la conquête de Grenade faisant passer ce dernier chiffre à 18 - que la Corte, c'est-à-dire la Cour, tend comme à avaler pendant le règne de Jean II (M. AsENJO GonZÁLEZ, « Ciudades y poder regio en la Castilla Trastámara (1400-1450) », dans Coups d'État à la fin du Moyen Âge?, op. cit., p. 380-384).

36. Outre les indications de l'édition employée, voir F. Gómez Redondo, Historia de la prosa, op. cit., t. III : Los orígenes del humanismo. El marco cultural de Enrique III y Juan II, Madrid, 2002, p. 2935-2943.

37. Frère Juan envisage tous les types de gouvernants (« régisseur », «gouverneur », «prince », « seigneur de royaume ou de terre», «principal du peuple»), quelle que soit la nature de leur pouvoir («gouvernement», «pouvoir», « seigneurie», « richesse», «privauté de roi »).

38. Libro del regimiento, op. cit., I.3, p. 241-242. Ce traité est à inscrire dans l'important débat sur le libre-arbitre et la prédestination qui marque la première moitié du Xv $\mathrm{Xv}^{\mathrm{e}}$ siècle (F. GómEZ ReDOnDO, Historia de la prosa, op. cit., t. III, p. 2277-2811).

39. C. Olivera SerRano, Las Cortes de Castilla y León y la crisis del reino (1445-1474). El registro de Cortes, Madrid, 1986, p. 13.

40. Sur la pénétration nobiliaire, voir mes récentes analyses dans « Patronazgo, relación de clientela y estructura clientelar. El testimonio del epílogo de la Historia de don Álvaro de 
Remarquons que dans le cadre de cette politique, ce prédicateur de cour qu'est probablement devenu frère Juan dès son retour d'Italie à la fin des années 1420 présente un profil qui le prédispose d'une certaine manière à la synthèse. Issu d'un lignage de la noblesse seconde et entré tardivement dans l'ordre augustin, sa formation le conduit en effet du convent de Valladolid au couvent Santo Spirito de Florence, et sans doute n'a-t-il pas manqué alors, en plus de passer sa licence, de parfaire sa connaissance des sociétés urbaines et de l'idéologie de leurs élites gouvernantes.

C'est en écho avec cette idéologie dont les maîtres mots sont le bien commun, l'utilité publique et la compétence, que frère Juan emploie le conseil de Jéthro dans son Libro del regimiento de los señores. Il le fait d'abord pour convaincre le gouvernant de prendre du repos, en désignant des remplaçants qui lui permettront de ne s'occuper que des affaires supérieures; et encore pourra-t-il, quand il voudra juger lui-même, en prendre connaissance au moyen de leur résumé (suma relación) ${ }^{41}$. Quant au second emploi, il sert à exhorter le prince à privilégier le «jugement de raison » plutôt que la « charnelle affection » ou la simple faveur quand il confie « à d'autres une partie de ses tâches » ${ }^{42}$. Autrement dit, dans la continuité de l'emploi de 1385 , le conseil de Jéthro sert ici à poser le principe d'une délégation gouvernementale destinée à rendre le gouvernement à la fois moins éreintant et plus efficace, ainsi que les critères permettant de bien choisir ceux qui doivent l'assumer.

Or, la convergence entre les développements de frère Juan et les exigences formulées au même moment par les représentants des villes aux Cortes est remarquable. Ainsi, les résumés que frère Juan préconise pour accélérer l'examen des affaires quand le gouvernant souhaite les juger par lui-même, mais qu'il envisage de manière très rigoriste afin d'installer comme un homme nu face au juge, font-ils l'objet de l'attention de la représentation urbaine entre 1436 et 1438 , celle-ci les trouvant trop brefs et réclamant pour les parties de pouvoir en prendre connaissance afin d'en vérifier le contenu ${ }^{43}$. Lorsqu'il dresse ensuite le profil de ceux que le prince doit choisir pour examiner à sa place les affaires ou encore prendre en main les gouvernements locaux, frère Juan ajoute aux considérations morales qui le portent à donner la préférence à des hommes mûrs ${ }^{44}$, des préoccupations relatives à leur idonéité, lesquelles révèlent l'importance qu'il accorde à la technicité et à l'expérience, disons de manière plus globale à la compétence. Ainsi

Luna », Hispania (à paraître). Sur la promotion des letrados pendant la privanza d'Álvaro de Luna, voir N. Round, The Greatest Man Uncrowned. A Study of the Fall of Don Álvaro de Luna, Londres, 1986, p. 169-176.

41. Libro del regimiento, op. cit., I.8, p. 259-260.

42. Ibid., III.4, p. 307-309.

43. Tolède, 1436, et Madrigal, 1438, CLC, III, p. 265 et 325-326.

44. Libro del regimiento, op. cit., I.8, p. 261-262. 
n'hésite-il pas à user de l'autorité d'Aristote et de l'image nautique pour légitimer la place de spécialistes dans le gouvernement royal ${ }^{45}$; et il affirme que les rois agissent finalement par amour, comme de bons pères, quand ils nomment des gouverneurs « lettrés, pieux et éprouvés » auxquels les nobles villes se fient ${ }^{46}$. Dans un cas comme dans l'autre, cette idée de compétence jette un sérieux doute sur la capacité « naturelle » à gouverner dont seraient pourvus les parents du roi et la noblesse. Et l'on sent bien ici à quel point frère Juan tente de ruiner certains des arguments employés par les Infants d'Aragon et leurs partisans contre le gouvernement du connétable, un parvenu à leurs yeux ${ }^{47}$.

Cette position méritocratique, quelque peu médiocratique aussi, que certains universitaires portés par le débat sur le gouvernement préférable accentueront bien plus nettement par la suite ${ }^{48}$, ne peut que satisfaire les représentants des villes. Car ils n'ont de cesse de réclamer, à l'inverse de ces «hommes de Palais qui savent mieux user de leurs armes que lire les livres des fors et des droits », qu'on leur donne pour les gouverner, des « hommes du commun [...] convenables pour cela » ${ }^{49}$, un gouverneur qui soit « idoine et convenable sans soupçon et du commun » ${ }^{50}$, aimant le «bien de la république ${ }^{51}$ plutôt que de ne penser qu'à s'enrichir et à semer la zizanie entre les peuples ${ }^{52}$. Enfin, une dernière convergence se fait jour entre la très forte réserve qu'émet frère Juan à propos des octrois de gouvernements urbains ou de seigneuries pour récompenser parents et obligés, qui soumettent les populations concernées à la menace d'une dévoration proprement tyrannique ${ }^{53}$, et l'itération au cours des années 1420-1430 des plaintes urbaines relatives à la démesure de la largesse royale et à la constance d'une

45. Cette image nautique sert à souligner quel esprit de corps doit unir le roi et ses officiers dans l'administration de la justice dans les Siete Partidas (II, 9.27). Selon le glosateur Gregorio López (glose h), cette métaphore nautique est tirée de l'Exameron de saint Ambroise (II.5). Elle est également employée par saint Thomas dans son De regno (I, 1.2, 9.30, 3.43 et 45), Georges Martin estimant possible qu'il ait pu avoir connaissance du code alphonsin («Alphonse X, roi et empereur (Commentaire du premier titre de la Deuxième partie) », Imprévue, 1-2, 1998, p. 23-54).

46. Libro del regimiento, op. cit., II.2, p. 281.

47. Voir notamment l'échange épistolaire de février-mars 1439 entre les partisans des Infants et le roi (Pedro CARrillo de Huete, Crónica del halconero de Juan II, J. de M. CARRIAZO éd., Madrid, 1946, p. 257-260 et 263-276).

48. J. L. Castillo Vegas, Política y clases medias. El siglo XV y el maestro salmantino Fernando de Roa ; A. RUCQUOI, « Démocratie ou monarchie Le discours politique dans l'université castillane au $\mathrm{XV}^{\mathrm{e}}$ siècle », dans El discurso político en la Edad Media, A. RUCQUOI et N. Guglielmi dir., Buenos Aires, 1995, p. 233-255.

49. Toro, 1371, CLC, II, p. 203-204.

50. Ocaña, 1422, CLC, III, p. 38.

51. Burgos, 1430, ibid., p. 92.

52. Zamora, 1432, ibid., p. 126.

53. Libro del regimiento, op. cit., III.4, p. 308. 
pression seigneuriale de ce fait accrue ${ }^{54}$, qui empêche finalement des villes ainsi détachée du domaine royal d'adresser des appels à la Cour ${ }^{55}$.

À ce point de convergence, le raisonnement de frère Juan devient trop distant par rapport à la réalité des pratiques gouvernementales et clientélistes de don Álvaro de Luna lui-même, principal courtier de la faveur royale. Aussi fait-il un peu machine arrière, en concédant finalement au prince de pouvoir remettre le gouvernement entre les mains de «parents familiers ou [de] loyaux serviteurs et vassaux » s'il prend soin préalablement de s'assurer de leur vertu et de les avertir de la lourde responsabilité que cet honneur représente. L'aveu d'insuffisance de la part de Moïse, puis sa peur d'assumer les souffrances du gouvernement, permettent à frère Juan d'ébaucher le principe d'une conduite idéale, très peu contemporaine cependant: un gouvernant n'assumant sa charge que le cœur gros, parce qu'il la sait énorme, accablante $^{56}$. Ce faisant, frère Juan fixe pour ces gouvernants en second, dont il légitime lui aussi la désignation en employant le conseil de Jéthro, un objectif d'excellence gouvernementale tout empreint de responsabilité et d'abnégation. Et le potentiel de vertus - jugement, discrétion, prudence et force - qu'il dit avoir senti dès sa dédicace chez le connétable de Castille ${ }^{57}$, qu'il entreprend par ce traité de lui faire découvrir afin de l'amener à devenir ce que Dieu a vraiment voulu qu'il soit, fait à n'en pas douter de celui-ci le meilleur des gouvernants possibles ${ }^{58}$. Dans un contexte, celui de la fin des années 1430, où se pose à nouveau la question du retour aux affaires des parents du roi, ce potentiel d'excellence que frère Juan attribue à son patron est un argument supplémentaire en faveur de cet abandon gouvernemental, jugé sans précédent et parfois mystérieux par ses détracteurs, que Jean II pratique en sa personne, parce qu'il est au fond la clef de sa plénitude souveraine ${ }^{59}$.

\section{La récupération}

Après l'exacerbation du régime de privanza sous le gouvernement luniste, Henri IV l'engage dans une voie de stabilisation contractuelle qu'une partie de l'aristocratie fait avorter, le laissant ainsi au bord de l'institution ${ }^{60}$.

54. Palenzuela, 1425 (pétition 13); Burgos, 1430 (pét. 16) ; Zamora, 1432 (pét. 12); Madrid, 1433 (pét. 9) ; Madrid, 1435 (pét. 15, 23, 28 et 33), Tolède, 1436 (pét. 8) ; Madrigal, 1438 (pét. 7, 53 et 54) dans CLC, III.

55. Valladolid, 1442, CLC, III, p. 428-429.

56. Libro del regimiento, op. cit., III.4, p. 308-309.

57. Ibid., p. 231.

58. Ibid., p. 195-213.

59. G. Agamben, Homo Sacer I. Le pouvoir souverain et la vie nue, Paris, 1997, p. 55-56.

60. F. FORONDA, «Vers un gouvernement de jure dans la Castille du $\mathrm{XV}^{\mathrm{e}}$ siècle : les contrats de privanza d'Henri IV de Trastamare », dans Du contrat d'alliance au contrat politique. La péninsule Ibérique à la fin du Moyen Âge, F. Foronda et A. I. CARrasco Manchado dir., Toulouse, 2007, p. 185-244. 
Et après le schisme monarchique et la guerre civile (1465-1468), la réconciliation et la pacification en passent par une réunion des Cortes. Aux villes qui répondent à l'appel et siègent de janvier à avril 1469 à Ocaña ${ }^{61}$, le roi fait part de son affliction et leur demande qu'elles l'aident à remédier aux maux du passé ${ }^{62}$. Mais si elles acceptent de collaborer, c'est qu'elles entendent cette fois s'entremettre dans les grandes affaires ou dans les corps de l'État - l'Audience et le Conseil - et du royaume, ce corps meurtri dont le roi n'a pas toujours su prendre soin selon leurs représentants ${ }^{63}$. Après s'être concertés ${ }^{64}$, ceux-ci parlent d'une même voix, produisant pour l'occasion un sermon dans la stricte continuité de leur essai en ce sens près de trente ans auparavant ${ }^{65}$. Un siècle après son invention de 1385 , le conseil de Jéthro fait ainsi son retour sur la scène parlementaire ${ }^{66}$.

De leurs attentes, les représentants ne doutent pas que le roi, en raison de son expérience et de ses lectures, disons plus généralement de sa prudentia regnativa, n'en ait déjà quelque véritable information ${ }^{67}$. Toutefois, parce qu'ils sont méfiants, ils préfèrent s'exprimer aussi clairement que possible, et rappellent quelques fondamentaux du métier royal ${ }^{68}$. Dans ce dessein, ils reprennent saint Thomas (De regno $\mathrm{I}, 1,2$ ) pour l'informer que son pouvoir procède de la naturalis necessitas, en tant qu'il est immanent à un corps social qu'il se doit de convertir en une multitudo consociata ${ }^{69}$. Ce n'est

61. Il manque alors les villes andalouses (Séville, Cordoue et Jaén) ainsi que Murcie, Tolède et Guadalajara (C. Olivera Serrano, Las Cortes de Castilla y León, op. cit., p. 122152).

62. Ocaña, 1469, CLC, III, p. 766-767.

63. Ce propos conduit les représentants à abandonner la distinction qu'ils établissent de manière réitérée depuis 1440 entre le service royal et le bien commun, pour différencier des domaines d'intervention, disons central et local, mais tenter de la sorte d'intervenir dans chacun (Valladolid, 1440 ; Burgos, 1453 ; Cordoue, 1455 ; Tolède 1462 ; CLC, III, p. 389, 642-643, 676-677 et 701-702).

64. Ocaña, 1469, ibid., p. 767.

65. D'un point de vue discursif en effet, il faut rapprocher la remontrance de 1469 de la pétition par laquelle les représentants des villes réclament à Jean II d'œuvrer à la pacification du royaume en 1440. À cette occasion, ceux-ci, bon thomistes, rappellent au roi qu'il doit être un agent de cohésion. Sans entrer dans le détail, ils poursuivent leur raisonnement en arguant des lois, de l'histoire, du sens commun ainsi que d'une expérience étrangère, celle du triste état dans lequel se trouve le royaume de France. Enfin, les représentants profitent de la semaine sainte qui les réunit pour demander au roi de suivre les pas du Christ, de son incarnation plus précisément, et qu'il s'abaisse donc à son tour pour procurer la paix à son peuple (Valladolid, 1440, CLC, III, p. 369-371).

66. Ibid., p. 767-769.

67. Selon saint Thomas, la prudentia regnativa fait appel à l'ensemble des facultés intellectuelles, en particulier le souvenir et la capacité de déduction (M. SENELLART, Les arts de gouverner. Du regimen médiéval au concept de gouvernement, Paris, 1995, p. 158-179).

68. La méfiance s'exprime ouvertement quand les représentants déclarent que le « désir de la plus grande partie de tous vos sujets et naturels est principalement de connaître par œuvre ce qu'il nous fut dit de votre part» (Ocaña, 1469, CLC, III, p. 766).

69. Sur la naturalis necessitas, voir M. SENELLART, Les arts de gouverner, op. cit., p. 162169. 
qu'après, comme saint Thomas, que les représentants usent des formules de saint Isidore, rex a regendo et recte agendo (Etymologiae IX, 3 ; Sententiae III, 48, 7), pour inscrire le métier de roi dans l'ordre éthique du regimen ${ }^{70}$. De là, il revient au roi de déterminer les quistiones (sic), de donner à chacun ce qui lui appartient, soit de rendre justice; obligation que les villes rappellent au roi depuis un certain temps déjà ${ }^{71}$. Le roi juge, et c'est parce qu'il juge qu'il est roi selon le Décret, que Dieu le maintient dans sa royauté conformément à la promesse faite à David (Ps 132, 11-12), pour qu'il accomplisse cette justice qu'il lui confie en le faisant régner $(\operatorname{Pr} 8,15)^{72}$.

Après cette mise au point, les représentants tirent une conclusion plus surprenante, en tout cas encore jamais énoncée aux Cortes : le roi n'est qu'un mercenaire, payé pour veiller sur son peuple pendant qu'il dort, un simple contractuel donc, obligé de servir cette communauté politique que les représentants estiment à présent représenter ${ }^{73}$. Ont-ils alors en tête les considérations d'Albert le Grand ou de Balde sur le rex exsomis et vigilans ${ }^{74}$; pensent-ils à ces nuits sans sommeil qu'Álvaro Pelayo attribuait à Alphonse XI pour glorifier le combat qu'il livrait contre les maures ${ }^{75}$; ou se souviennentils simplement d'un autre psaume : «il ne sommeille ni ne dort celui qui garde Israël » (Ps 121, 4) ? En tout cas, les représentants prient le roi qu'il veuille bien s'en tenir au contrat tacite (contrato callado) qui le lie à ses sujets, en vertu duquel il perçoit une part des fruits de leur industrie, contre l'obligation de les « maintenir en justice ${ }^{76}$.

Ensuite, les représentants invitent le roi à faire enfin le Roi, en lui rappelant la paire primordiale du modus agendi monarchique que sont la miséricorde et la justice ${ }^{77}$, puis en lui proposant de méditer l'exemple de Trajan sauvé par saint Grégoire des peines de l'Enfer (Jean Diacre, Sancti Gregori Magni Vita II, 44 ; Jean de Salibury, Policraticus V, 8). C'est alors qu'ils reprennent le conseil de Jéthro. Mais ils s'avèrent meilleurs sermo-

70. Ibid., p. 65-90 ; Ph. Buc, L’ambiguïté du Livre, p. 258-272.

71. Par exemple Valladolid, 1447 ; Burgos, 1453 ; CLC, III, p. 524 et 642. Dans le deuxième cas, le rappel de l'obligation donne lieu à l'emploi de la métaphore organiciste.

72. La référence au décret semble ici plutôt générique. Elle renvoie à la question du roi comme iustitia et lex animata, une idée qui s'exprimait déjà, en 1442, quand les représentants rappelaient que la loi écrite reste comme lettre morte si la loi vivante ne vient pas la défendre ni lui donner vigueur (Valladolid, 1442, ibid., p. 444). Sur la question, voir mes précédents commentaires dans F. FORONDA, «La privanza, entre monarquía y nobleza », dans La monarquía como conflicto, J. M. NIETO SoRIA dir., op. cit., p. 121-124.

73. R. Morán Martín, loc. cit.

74. E. Kantorowicz, Les Deux Corps du Roi. Essai sur la théologie politique au Moyen Âge, Paris, 1989, p. 422, 424 et 427-428.

75. Álvaro Pais, Espelho dos reis, M. Pinto DE Meneses éd., Lisbonne, 1955, t. I, p. $22-23$.

76. Bien que débarrassée des images traditionnelles de la tête, du cœur ou encore de l'estomac, la conception qu'ont les représentants des villes du contrat tacite relève bien d'une conception organiciste de la communauté politique.

77. P. Buc, «Pouvoir royal et commentaires de la Bible (1150-1350) », Annales ESC, 3, 1989, p. 691-713. 
neurs qu'archivistes, car ils le pensent lié à la création de l'Audience par Henri II en 1371. Le déplacement n'est pas fortuit bien entendu. Dans l'esprit des représentants, c'est bien à l'Audience que revient l'administration de la justice et non pas au Conseil qu'ils estiment dominé par les Grands ${ }^{78}$. C'est donc une autre conception de la hiérarchie entre les corps de l'État qui s'exprime. Davantage encore, en repoussant l'invention du conseil de Jéthro au temps d'Henri II, les représentants lui donnent la valeur d'un pacte fondateur, pas vraiment respecté par les Trastamare comme le montre la ruine de cette « maison de justice » qu'est l'Audience, conçue selon eux sur le modèle du Sacré Collège.

Rappel à l'ordre quelque peu cinglant, le sermon des représentants se veut aussi le premier acte d'une restauration, d'une réédification à partir des sept piliers de Sagesse qu'ils considèrent encore intacts $(\operatorname{Pr} 9,1)$ et dont ils se veulent les gardiens ${ }^{79}$. De là, le conseil de Jéthro pour dire au roi, encore, qu'il doit choisir pour gouverner des « hommes prudents, qui craignent Dieu, qui soient sages et aient en horreur l'avarice », à l'inverse de ces « ministres idiots ou malicieux » qui ont provoqué la ruine de la «maison de justice ». Ce n'est donc pas le principe d'une délégation gouvernementale qui est remis en cause, mais seulement ses bénéficiaires. Pour finir, les représentants se font un peu rassurants. Ils invitent le roi au seul amour véritable, celui de sa justice, et lui disent qu'il pourra entendre, alors, résonner de nouveau la voix du Prophète : «Tu aimes la justice, tu hais l'impiété. C'est pourquoi Dieu, ton Dieu, t'a donné l'onction d'une huile d'allégresse comme à nul de tes rivaux » (Ps 45, 8).

Des auteurs de cette ultime promesse de félicité, les actes des Cortes ne disent rien. Mais tant de ressorts d'un langage également utilisé par la Chancellerie royale depuis le XIV ${ }^{\mathrm{e}}$ siècle confirment le rapprochement, largement orchestré depuis le trône même, entre les représentants des villes et les officiers du roi, et plus particulièrement parmi ces derniers de ceux que la désertion courtisane de certains Grands pendant la guerre civile a propulsé sur le devant de la scène. De fait, l'arsenal référentiel activé par la remontrance d'Ocaña n'est pas sans rappeler celui dont use l'un d'eux dans sa chronique du règne, Diego Enríquez del Castillo. Après des études en théologie à l'Université de Salamanque, le licencié Enríquez del Castillo trouve place dans la Chapelle princière puis royale d'Henri IV. En 1460, le roi le nomme son chroniqueur puis l'admet à son Conseil, sans doute autour de

78. Là encore, la remontrance de 1469 se situe dans le prolongement des revendications des années 1430-1440, en particulier de la pétition présentée à Jean II aux Cortes de Valladolid pour qu'il remette l'ensemble des cas civils et criminels à l'Audience et à la Chancellerie plutôt qu'au Conseil (Valladolid, 1440, CLC, III, p. 383).

79. Ce devoir de défense s'exprime au même moment dans le discours produit par les fraternités et milices urbaines (J. L. BERMEJo CABRERO, « Hermandades y comunidades de Castilla », Anuario de Historia del Derecho Español, 58, 1988, p. 277-412 ; J. M. NiETo SoRIA, «Fragmentos de ideología política urbana en la Castilla bajomedieval », Historia medieval. Anales de la Universidad de Alicante, 13, 2000-2002, p. 203-229). 
1461-1462, dans le sillage de don Beltrán de la Cueva et des Mendoza. Au cours de la guerre civile, Enríquez del Castillo reste fidèle à Henri IV et œuvre à renforcer le parti légitimiste, comme en témoignent ses relations privilégiées avec la milice populaire que forme l'Hermandad General. Ainsi est-il chargé par Henri IV de rédiger le sermon qu'il adresse par lettre à la junte générale de Tordesillas en $1466^{80}$. Porte-parole du roi auprès de l'Hermandad, Diego Enríquez l'est aussi de celle-ci auprès d'Henri IV l'année suivante, afin de le détourner du plan de pacification que promeut l'archevêque Fonseca. Et face au refus d'Henri IV, son chroniqueur et quelques autres, dont les capitaines de l'Hermandad, soulèvent le peuple madrilène afin d'empêcher son départ ${ }^{81}$.

Pour ce groupe de «loyaux serviteurs », dominé par les officiers du roi mais en étroite relation avec l'Hermandad, la libération préventive de Madrid est sans nul doute une façon de bloquer un rapprochement dont ils craignent de faire les frais ${ }^{82}$. Remarquons d'ailleurs que Rodrigo de Morales, membre de l'oligarchie de Soria, également vassal d'Henri IV, très lié aux Mendoza et accusé avec Enríquez del Castillo et d'autres par le chroniqueur alphonsiste Alfonso de Palencia d'être à l'origine du soulèvement madrilène de $1467^{83}$, prend part aux Cortes de 1469 en qualité de représentant de Soria ${ }^{84}$. Aussi le rebond critique d'origine urbaine du conseil de Jéthro n'est-il possible que parce qu'il renferme une sorte de fronde des Petits ou des Moyens de l'État monarchique, que le plein retour aux affaires des Grands, les anciens « serviteurs spéciaux » des contrats de privanza d'avant la guerre civile, menace de relégation politique. Contraints par cette convergence d'intérêts, les Grands se montrent conciliants. Le 25 avril, ils s'engagent à ce que le roi réponde à toutes les pétitions, et consentent à ce que certains des représentants prennent place, à leur côté, à la table du Conseil ${ }^{85}$.

Mais d'un point de vue discursif, la réponse au rebond critique d'Ocaña n'intervient que sept ans plus tard. Elle est donnée cette fois par Isabelle et Ferdinand aux Cortes de Madrigal, convoquées dans la certitude d'une victoire déjà presque totale dans la guerre de succession ${ }^{86}$. Cette

80. Diego Enríquez del Castillo, Crónica de Enrique IV, A. Sánchez Martín éd., Valladolid, 1994, p. 260-262.

81. Ibid., p. 267-270.

82. F. FORONDA, «S'emparer du roi. Un rituel d'intégration politique dans la Castille trastamare », dans Coups d'États à la fin du Moyen Âge?, op. cit., p. 213-329.

83. Alfonso de Palencia, Gesta hispaniensia ex annalibvs svorvm diervm collecta, B. Tate et J. Lawrance éd., Madrid, 1999, t. II, p. 412.

84. C. Olivera Serrano, Las Cortes de Castilla y León, op. cit., p. 148 ; M. Diago Hernando, Estructuras de poder en Soria a fines de la Edad Media, Valladolid, 1993, p. 84, 93, 99, 168, 178, 194, 198, 233, 275 et 276.

85. C. Olivera Serrano, Las Cortes de Castilla y León, op. cit., doc. 65 et 66, p. $332-$ 335 ; L. SuÁrez FERnÁNDEZ, Enrique IV de Castilla. La difamación como arma política, Barcelone, 2001, p. 420-427.

86. J. M. Carretero Zamora, Cortes, Monarquía, Ciudades. Las Cortes de Castilla a comienzos de la época moderna (1476-1515), Madrid, 1988, p. 131-141. 
réponse prend la forme d'une pastorale monarchique fondée sur la grâce ${ }^{87}$, probablement en partie tributaire du rigorisme responsabilisant de fray Juan de Alarcón ${ }^{88}$. Et si cette grâce responsabilise absolument le roi de son gouvernement devant Dieu, il se trouve obligé à la performance s'il veut actualiser la relation d'amour qui le lie à son créateur, depuis la «profession et état » qu'il lui assigne ${ }^{89}$. Remarquons que si la communauté politique se trouve également engagée dans un gouvernement ainsi transformé en action de grâce, de ce fait peut-être dépolitisé, elle l'est de manière atomisée, chacun de ses membres se voyant rivé à sa «profession et état », ce qui revient à dissiper toute idée de contrat, même tacite, entre cette communauté et son gouvernant.

Au total, le conseil de Jéthro permet d'envisager un moment de la communication entre le roi et les villes, sur la base d'un même langage, celui de la théologie politique. Le maniement de ce langage partagé signale indéniablement une intégration politique plus poussée. Ainsi, ce conseil de Jéthro révèle-t-il un partage de pouvoir autant qu'il le nourrit. Mais son sens se fait plus équivoque depuis la perspective de l'élargissement de la société politique. Car le rebond critique de 1469 est la conséquence d'un important resserrement de la représentation urbaine à partir de la fin du XIV siècle et de l'investissement de celle-ci par les officiers du roi au cours du XV siècle. C'est donc comme une communication progressivement internalisée, dans l'État, que donne principalement à voir l'histoire de ce fragment de théologie politique, parce qu'il sert précisément à ouvrir son gouvernement en légitimant sa délégation, laquelle contribue à étatiser la société. Il y a là comme le principe d'une institution politique que la remémoration du conseil de Jéthro tend à entretenir ${ }^{90}$, comme le montre plus particulièrement l'erreur de datation commise par les représentants des villes à Ocaña. D'autres ajustements invitent à pousser cette idée d'entretien en direction d'une sacralisation qui vise à rendre intouchable le pacte fondateur enfin explicité. Ainsi frère Juan hésite à voir dans le partage gouvernemental institué par Moïse l'exécution d'un commandement divin ou la mise en pratique du conseil donné par Jéthro. À la veille de cette nouvelle crise politique que sont les

87. Madrigal, 1476, CLC, IV, p. 1-2. Le thème du préambule de l'Ordenamiento est donné par l'épître aux Romains de saint Paul (Rm 8. 28 et 12), référence que complètent ensuite une mention de l'évangile selon saint Luc (12.48), une autre des Proverbes (8.12, également présente dans la remontrance d'Ocaña) et une autre enfin du Livre de la Sagesse (1.1).

88. La transmission entre fray Juan et Isabelle la Catholique se fait principalement par le biais d'un autre observantin au service d'Álvaro de Luna, frère Martín de Córdoba, qui lui dédie en 1468 un miroir au prince au féminin, le Jardín de las nobles donzellas (F. GómEZ REDONDO, Historia de la prosa, op. cit., t. IV : El reinado de Enrique IV : el final de la Edad Media, Madrid, 2007, p. 3661-3677). 1996.

89. B. Clavero, La grâce du don. Anthropologie catholique de l'économie moderne, Paris,

90. F. Ost, Raconter la loi, op. cit., p. 18-19. 
Comunidades, lors des Cortes de Valladolid de 1518, les représentants des villes reprennent une partie de la remontrance d'Ocaña de 1469 et tranchent en faveur d'un commandement divin ${ }^{91}$.

François Foronda, Université Paris I Panthéon-Sorbonne, Laboratoire de Médiévistique Occidentale de Paris (UMR 8589), 17 rue de la Sorbonne, F-75005 Paris, Grupo Consolidado de Investigación UCM 930369

\section{Le conseil de Jéthro à Moïse : le rebond d'un fragment de théologie politique dans la rhétorique parlementaire castillane}

L'analyse du rebond du conseil de Jéthro à Moïse (Ex. 18, 13-27) dans la rhétorique parlementaire castillane entre 1385 et 1469 est l'occasion d'étudier un moment de la communication politique entre le roi et les villes. Leur maniement d'un même langage signale un processus d'alphabétisation politique et d'intégration gouvernementale. Ainsi, ce fragment de théologie politique se transforme-t-il en récit fondateur d'un gouvernement en partage.

Castille - communication politique - rhétorique parlementaire - récit fondateur - gouvernement partagé.

\section{Moses'Advice to Jethro : Reviving a Fragment of Political Theology in Castilian Parliamentary Rhetoric}

The analysis of the use of the council of Jethro to Moses (Ex. $18: 13-27)$ in the parliamentary rhetoric in Castile between 1385 and 1469, is an opportunity to study a moment of political communication between king and towns. Their use of a same language highlights a process of political alphabetisation and of governmental integration. Thus, this fragment of political theology is transforming in a foundational narrative of a shared government.

Castile - political communication - parliamentary rhetoric - foundational narrative - shared government

91. Valladolid, 1518, CLC, IV, p. 262 (repris dans R. MoRÁn MARTín, « alteza... merçenario soys », loc. cit.). 